\title{
Are Women Aware of the High Prevalence Rates of Anorectal Dysfunction After Childbirth?
}

\author{
Neels $\mathbf{H}^{1,4^{*}}$, Tjalma WAA ${ }^{2,4}$, De Wachter $\mathbf{S}^{3,4}$, Wyndaele $\mathbf{J J}^{3}$ and Vermandel $\mathbf{A}^{1,4}$ \\ ${ }^{1}$ Department of Rehabilitation Sciences and Physiotherapy, Faculty of Medicine and Health Sciences, University of Antwerp, Belgium \\ ${ }^{2}$ Department of Gynecologic Oncology, Faculty of Medicine and Health Sciences, Antwerp University Hospital, University of Antwerp, Belgium \\ ${ }^{3}$ Department of Urology, Faculty of Medicine and Health Sciences, Antwerp University Hospital, University of Antwerp, Belgium \\ ${ }^{4}$ Antwerp University Hospital, Belgium
}

*Corresponding author: Hedwig Neels, Faculty of Medicine and Health Sciences, Department of Rehabilitation Sciences and Physiotherapy, Revaki Campus Drie Eiken-Lokaal, University of Antwerp, Belgium, Tel: +32 326528 59; E-mail: hedwig.neels@uantwerpen.be

Received date: March 27, 2017; Accepted date: July 20, 2017; Published date: August 11, 2017

Copyright: (c) 2017 Neels H, et al. This is an open-access article distributed under the terms of the Creative Commons Attribution License, which permits unrestricted use, distribution, and reproduction in any medium, provided the original author and source are credited.

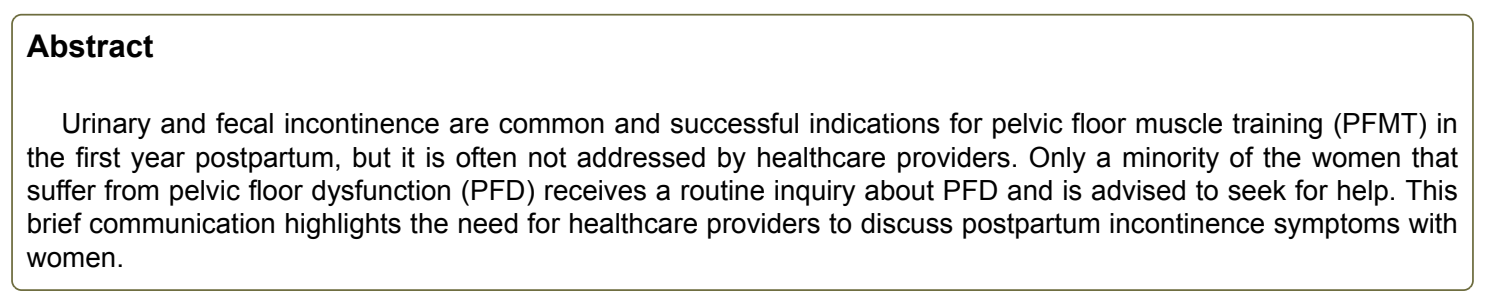

Keywords: Anal incontinence; Childbirth; Episiotomy; Fecal urgency; Vaginal delivery; Knowledge; Pelvic floor muscle training

\section{Discussion}

Pregnancy and childbirth are the most important risk factors that contribute to PFD. Disorders of defecation are undoubtedly the most severe adverse events of PFD associated with childbirth. Anal incontinence (AI), the involuntary loss of flatus, liquid, or solid stool, is a distressing and debilitating condition with considerable impact on occupational, social, and sexual quality of life. Rusavy et al. investigated AI following mediolateral $(n=300)$ or lateral $(n=360)$ episiotomy during a first vaginal delivery [1].

Their study showed AI among 7 and $3 \%$ of patients who underwent mediolateral and 7 and $6 \%$ of who underwent lateral episiotomy, at respectively 3 and 6 months postpartum. They concluded that AI was comparable between both groups and that the association between lateral episiotomy and fecal urgency merits further scientific interest. Next to the high prevalence of AI after childbirth in primiparous women.

Previous research has showed that nulliparous $(n=212)$ and primigravid $(n=221)$ women have a significant lack of knowledge about the pelvic floor and the risk of PFD postpartum $[2,3]$.

Table 1 shows that the risk of urinary incontinence after delivery is known by most women; but disorders of defecation and flatulence were only known by a minority. The majority of the nulliparous and primigravid women never receive any information about PFD. But almost all women confirm that they are interested to receive more information (Table 1) [2,3].

\begin{tabular}{|c|c|c|c|c|}
\hline \multicolumn{5}{|c|}{ How much do you know about the pelvic floor muscles on a scale from zero to ten, whereas zero is absolutely nothing and ten is expert in the domain? } \\
\hline PG (221) & \multicolumn{4}{|c|}{3.7 to 10 (SD 2.59) } \\
\hline \multicolumn{5}{|c|}{ Does a healthy woman occasionally lose stool, immediately after delivery, 1 month after delivery and 6 months after delivery? } \\
\hline 1-5 d PP & NP (212) & $51(24 \%)$ Yes; 85 (40\%) I don’t know & PG (221) & 34 (15\%) Yes; 63 (29\%) I don't know \\
\hline $1 \mathrm{~m} \mathrm{PP}$ & NP (212) & 52 (25\%) Yes; 156 (74\%) I don't know & PG (221) & $2(1 \%)$ Yes; 61 (28\%) I don't know \\
\hline $6 \mathrm{~m} \mathrm{PP}$ & NP (212) & 45 (21\%) Yes; 165 (78\%) I don’t know & PG (221) & 1 (1\%) Yes; 55 (25\%) I don't know \\
\hline \multicolumn{5}{|c|}{ Which consequences can you expect after delivery? } \\
\hline UI & NP (184) & $147(80 \%)$ & PG (221) & $162(73 \%)$ \\
\hline Stool problems, Al & NP (185) & $26(14 \%)$ & PG (221) & $35(16 \%)$ \\
\hline Flatulence & NP (182) & $18(9 \%)$ & PG (221) & $16(7 \%)$ \\
\hline
\end{tabular}


Citation: Neels H, Tjalma WAA, De Wachter S, Wyndaele JJ, Vermandel A (2017) Are Women Aware of the High Prevalence Rates of Anorectal Dysfunction After Childbirth?. J Women's Health Care 6: 380. doi:10.4172/2167-0420.1000380

Page 2 of 2

\begin{tabular}{|c|c|c|c|c|}
\hline \multicolumn{5}{|c|}{ Experience with education and gathering of information on this topic } \\
\hline Ever received information? & NP (208) & $40(19 \%)$ Yes & PG (219) & $62(28 \%)$ Yes \\
\hline Sufficiently informed? & NP (205) & $15(7 \%)$ Yes & PG (221) & $178(81 \%)$ Yes \\
\hline Interested in more information? & NP (206) & $191(93 \%)$ Yes & $P G(221)$ & $195(88 \%)$ Yes \\
\hline
\end{tabular}

Table 1: Pelvic floor Knowledge of nulliparous women (nulliparous and primigravid group) [d: Days; m: Months; PP: Postpartum; SD: Standard Deviation; UI: Urinary Incontinence; AI: Anal Incontinence; NP: Nulliparous Women; PG: Primigravid Women].

The research about postpartum AI performed and the latest IUGA consensus about anorectal dysfunction in women are a significant aid to clinical practice and a stimulus for research. While the taboo about these PFD is clearly diminishing in scientific research, the performed research highlights the taboo and ignorance that prevails among women about AI [2,3]. Therefore, we emphasize the need of a good education about the pelvic floor and PFD in young women, before they are exposed to the greatest risk factors such as pregnancy and delivery. Education might offer the first way to reduce dysfunction by promoting help seeking behavior, prevention and treatment.

\section{Conflict of Interest}

The authors of this brief communication report no conflicts of interest.

\section{References}

1. Rusavy Z, Karbanova J, Jansova M, Kalis V (2016) Anal incontinence and fecal urgency following vaginal delivery with episiotomy among primiparous patients. Int J Gynaecol Obstet 135: 290-294.

2. Neels H, Wyndaele JJ, Tjalma WA, De Wachter S, Wyndaele M, et al. (2016) Knowledge of the pelvic floor in nulliparous women. J Physical Therapy Sci 28: 1524-1533.

3. Neels H, Tjalma WA, Wyndaele JJ, De Wachter S, Wyndaele M, et al. (2016) Knowledge of the pelvic floor in menopausal women and in peripartum women. J Physical Therapy Sci 28: 3020-3029. 